\title{
A Chance-Constrained Optimization Approach for Control of Transmission Voltages
}

\author{
Mahdi Hajian, Student Member, IEEE, Mevludin Glavic, Senior Member, IEEE, \\ William D. Rosehart, Senior Member, IEEE, and Hamidreza Zareipour, Senior Member, IEEE
}

\begin{abstract}
In this paper, a chance-constrained optimization (CCO) is presented to handle uncertainty in control of transmission voltages. A control scheme is proposed using a steady-state system model to achieve the goal of online voltage control and preventing long-term voltage instability. In order to model steady-state system response, the long-term model of governors and Automatic Voltage Regulators are employed in the control scheme. The Nordic32 test system is selected to show the simulation results of the proposed technique.
\end{abstract}

Index Terms-Chance-constrained optimization (CCO), long-term voltage instability, voltage control.

\section{INTRODUCTION}

T HE goal of voltage control is to keep system voltages within specified limits while accounting for associated limits such as generators reactive powers. After a large disturbance, power systems might face nonviable or unstable voltages, potentially leading to voltage instability that need to be corrected [1], [2]. An important issue in this regard is to ensure that, given anticipated uncertainty in system model, the control scheme results in control actions that lead to feasible operating conditions.

Online voltage control schemes, related to corrective actions, are addressed in several works. In [3], a receding horizon multistep optimization is proposed to control transmission voltages based on steady-state power flow equations. A coordinated secondary voltage control according to sensitivity analysis, operational in two French control centers, is presented in [4]. The feasibility of utilizing the French voltage control scheme for emergency actions, namely load shedding, is presented in [5]. A model predictive control (MPC) approach is used in [6], where the coordination of generator voltage settings, tap changers, and load shedding is presented and solved by the tree search optimization technique. Reference [7] studies a coordinated voltage control framework based on nonlinear system equations with Euler state prediction and pseudo gradient evolutionary programming.

Manuscript received August 08, 2011; revised October 04, 2011; accepted December 16, 2011. Date of publication February 03, 2012; date of current version July 18, 2012. Paper no. TPWRS-00742-2011.

M. Hajian, W. Rosehart, and H. Zareipour are with the Department of Electrical and Computer Engineering, Schulich School of Engineering, University of Calgary, Calgary, AB, Canada T2N 1N4 (e-mail: mhajian@ucalgary.ca; rosehart@ucalgary.ca; h.zareipour@ucalgary.ca).

M. Glavic is with Quanta Technology, Raleigh, NC 27607 USA, and also with the Department of Electrical Engineering and Computer Science (Montefiore Institute), University of Liege, B-4000 Liege, Belgium (e-mil: mglavic@quantatechnology.com).

Digital Object Identifier 10.1109/TPWRS.2011.2181431
The literature dealing with uncertainty in voltage control is mostly based on preventive actions, performed with offline analysis. In [8], a framework is proposed to minimize voltage stability L-index subject to contingencies where uncertainty in forced outage rate of generations and transmission lines are incorporated using fuzzy membership function. In [9], reactive power reserve is maximized based on static system equations using an improved particle swarm optimization, and then, given uncertainties in system parameters and variables, the probability of voltage collapse is estimated by a trained radial basis function network. In [10], a security constrained optimal reactive power planning is studied employing fuzzy sets to model uncertainty in demand. A sampling-based approach is proposed in [11] to evaluate voltage stability of series-compensated transmission lines taking into account uncertainty in load parameters. A fuzzy multi-objective algorithm is presented [12] to control of distribution voltages where uncertainty in active load power, load power factor, and source-node voltage is incorporated using membership functions. A stochastic evaluation of distribution voltage profiles with distributed generation (DG) is studied in [13] considering uncertainty in load, DG production, and network configuration. A probabilistic voltage stability analysis is presented in [14] using Monte Carlo simulation and D' matrix method where uncertainty in demand and equipment availability exists. In [15], the CCO approach to optimal power flow problem is demonstrated and the problem is solved considering individual chance constraints via linear approximation of power flow equations. In [16], the stochastic optimal reactive power dispatch is proposed and solved via CCO approach and genetic algorithm with the aid of a linear model based on sensitivity analysis.

In addition to $\mathrm{CCO}$, scenario-based techniques and two-stage recourse models are frequently used in stochastic optimizations. In the scenario-based technique, the optimization problem is solved for selected scenarios and then the weighted expected value of all solutions is normally calculated as the final solution [17]. In the two-stage recourse model, the solution is divided into first-stage decisions and second-stage decisions where firststage decisions are "here and now" policy (decision variables are decided before the actual realization of uncertain variables) and second-stage variables determine which action needs to be taken against each outcome of uncertain variables [18].

Incorporating uncertainty in online schemes is mainly studied in the control literature. In [19], an MPC problem is presented with linear inequality constraints and uncertain parameters, and solved with CCO. In [20], the real-time optimization with nonlinear constraints is studied and solved 
using joint chance constrained and an iterative method. A chance-constrained-based model predictive control method is used in [21] where time dependent uncertain variables are involved with corresponding joint distribution functions. In [22], a probabilistically constrained predictive control considering joint chanced constraint is proposed and solved by numerical integration.

In this paper, a $\mathrm{CCO}$ approach based on an online voltagecontrol scheme is proposed to correct unstable transmission voltage and mitigate long-term voltage instability considering uncertainties in the system model. The control actions are generators voltage reference and load shedding where uncertainty exists in the amount of load available for shedding. A steady-state nonlinear model is used to determine control actions needed for voltage correction, and verifying the feasibility of a derived solution given the existence of uncertainty. To this purpose, a steady-state model including governor responses, automatic voltage regulators (AVRs), and power-flow-based equations are employed in the control scheme. This paper addresses the shortcoming of single-step optimization (SSO) presented in [3] in handling model uncertainty. The SSO optimization problem in this paper is reformulated using the $\mathrm{CCO}$ approach where the uncertainty appears in the objective function and a set of constraints on the amount of load shedding. The optimization problem is solved using an iterative approach to satisfy the probability of intersection of probabilistic constraints. In [3], uncertainty is not directly considered. The CCO problem solved in this paper differs from the one presented in [15] and [16] proposed for optimal-power-flow-based problems in the sense of using a joint rather than an individual $\mathrm{CCO}$ and solving the nonlinear system model without the need to linearization. In addition, Latin hypercube sampling with Cholesky decomposition (LHS-CD) is utilized to generate efficient samples of the random variables required to derive the probability of feasibility of computed control actions.

The remainder of this paper is organized as follows. The general assumptions and theory behind the voltage control scheme and CCO is presented in Section II. The problem formulation of the proposed technique is demonstrated in Section III. Simulation results are presented in Section IV. Finally, Section V concludes the paper.

\section{ASSUMPTIONS AND BACKGROUND}

\section{A. Voltage Control}

The objective is to correct nonviable or unstable transmission voltages after an occurrence of a disturbance in the system. The focus is on long-term voltage stability where the actions of load tap changers (LTCs) and overexcitation limiters (OELs) are mostly involved in dynamic system evolution [2]. It is assumed that the snapshots of voltage measurements and generators active power injections are available with a sample period about $10 \mathrm{~s}$. This can be achieved via a wide-area monitoring (WAM) system which uses enhanced measurements provided by PMUs.

The control variables considered include generator voltage setpoints and load curtailment. The problem formulation allows using additional controls, such as transmission-line tap changers, active power of generators, and FACTS devices using their steady-state model. The actions of automatic LTCs is implicitly modeled in the controller utilizing the pre-disturbance load value calculated based on the last snapshot of measurements received by the controller before the disturbance. This is attributed to load power restoration which happens via the restoration of distribution voltages by LTCs, where it can be assumed that the load evolves to its pre-disturbance value [3].

The amount of load available for curtailment is considered to be a random variable modeled with a normal distribution. Although any distribution can be used in the proposed stochastic controller, the normal distribution is selected since it represents the distribution of demand in power systems [23]. It was assumed that the constant power factor is preserved, therefore, the same factor of uncertainty exist in the active and reactive power available for curtailment. The formulation used in this paper accommodates incorporating any uncertain parameter presented as a linear term in the mathematical representation of the problem.

\section{B. $C C O$}

Chance-constrained is a type of stochastic programming which incorporates randomness into the model via a probabilistic measure over uncertain constraints [24]. A typical CCO problem can be written as

$$
\begin{array}{ll} 
& \min _{\mathbf{y}} f(\mathbf{y}, \boldsymbol{\zeta}) \\
\text { s.t. } & h_{0}(\mathbf{y})=\operatorname{Pr}\left(g_{r}(\mathbf{y}, \boldsymbol{\zeta}) \geq 0 \quad r=1, \ldots, k\right) \geq \alpha \\
& h_{i}(\mathbf{y}) \geq p_{i} i=1, \ldots, m
\end{array}
$$

where $f(\mathbf{y}, \boldsymbol{\zeta})$ is the objective function which generally includes random variables, $\mathbf{y}$ is the vector of decision variables, and $\boldsymbol{\zeta}$ is the vector of $K$ random variables with given cumulative density functions (CDFs) such that $F_{\zeta_{j}}(z)=\operatorname{Pr}\left(\zeta_{j} \leq z\right)(j=$ $1, \ldots, K)$. The set of joint probabilistic constraints is described in (1b), where $\operatorname{Pr}()$ denotes the probability measure, $g_{1}, \ldots, g_{k}$ are the set of constraints which involve random variables, and $\alpha$ is the specified confidence level on the probabilistic constraint to be satisfied. Equation (1c) presents the set of deterministic constraints with constant lower bounds as $p_{1}, \ldots, p_{m}$.

The joint probability $\alpha$ is the probability of intersection of $k$ individual constraints to be satisfied. The solution to the joint $\mathrm{CCO}$ problem can be found directly using a multidimensional integral given the joint distribution of $\zeta$. However, this approach suffers from the problem of numerical processing of a multidimensional integration and the solution space not being convex [25]. Another approach is to convert the joint chance constraint to a set of individual chance constraint [24], [26], [27]. In the case that the function $g_{r}(\mathbf{y}, \boldsymbol{\zeta})$ can be linearly expressed in terms of $\zeta$ such that $g_{j}(\mathbf{y}, \boldsymbol{\zeta})=\sum_{i} T_{j i} y_{i}-\zeta_{j}, j=1, \ldots, K$, it can be shown that the set of equivalent individual chance constraints can be written as follows:

$$
\begin{aligned}
\sum_{i} T_{j i} y_{i} & \geq E\left(\zeta_{j}\right)+\sqrt{\operatorname{Var}\left(\zeta_{j}\right)} Z_{\alpha} \\
Z_{\alpha} & =\Phi^{-1}\left(1-\frac{1-\alpha}{K}\right)
\end{aligned}
$$


where $E()$ and $\operatorname{Var}()$ are the functions that return the expected value and variance of a random variable, and $\Phi$ is the standard normal CDF. Using the individual constraints (2) results in a more conservative solution than the joint chance constraint (1b). Therefore, it is required to update the value of $Z_{\alpha}$ in order to find a solution which satisfies the required confidence level. The method for updating $Z_{\alpha}$ proposed in [26] is used, which is based on the interpolation of $Z_{\alpha}$ values and discussed in more details in Section III-B.

The objective function can be transformed to an equivalent deterministic one employing the $E$-model, $V$-model, or $P$-model [24]. In this paper, the E-model approach is considered in which a linear objective function expressed as $f(\mathbf{y}, \boldsymbol{\zeta})=\zeta^{T} \mathbf{y}$ is replaced with $[E(\zeta)]^{T} \mathbf{y}$. This approach was used due to the observed behavior of the problem formulation resulting in objective values with an average close to the expectation and relatively low variations.

\section{Latin Hypercube Sampling With Cholesky Decomposition (LHS-CD)}

Traditionally, Monte Carlo simulation along with simple random sampling (SRS) is used in probabilistic problems in power systems such as probabilistic power flow [28], [29]. This method could provide highly accurate results given a sufficiently large sample size, however, the drawback is the computational burden associated with the large number of repeated calculations. Latin hypercube sampling (LHS) was first introduced in [30] and was shown to produce better results compared with the SRS method [31], [32]. In traditional LHS, denoted as LHS-RP, the run order of different samples is determined based on a random permutation for the number of samples. However, in multivariate probabilistic problems as the number of random variables increases, this method suffers from the lack of producing samples with minimum correlation among them. In this paper, LHS-CD is used which can generate samples of random numbers given any predefined correlation among the variables [33]. Given the characteristic of ordering matrix generated by LHS-RP being a positive definite matrix, Cholesky decomposition is employed in order to set the correlation among variables. In this paper, zero correlation among the uncertain variables is taken into account. The details on the algorithm to generate LHS-CD samples can be found in [32].

\section{CCO IN VOLTAGE CONTROL}

\section{A. Stochastic Optimization Formulation}

In this work, an extension of the SSO scheme presented in [3] is proposed to accommodate uncertainty in the model. In addition, it is proposed that steady-state equations can still be used in the control scheme to model the long-term behavior of system. The problem of correcting nonviable or unacceptable transmission voltages can be formulated as finding a new steady-state equilibrium point while minimizing changes in control variables. While accounting for uncertainty in system model, namely load available for shedding, the associated CCO-based OPF problem can be written as

$$
\begin{array}{ll} 
& \min _{\mathbf{u}_{\mathbf{r}}, \mathbf{u}_{\mathbf{p}}, \mathbf{x}} \sum_{i=1}^{n_{r}} c_{i}\left[u_{r i}{ }^{o}\left(0^{-}\right)-u_{r i}\right]^{2} \\
& +E\left(\sum_{i=1}^{n_{p}} c_{i}\left[u_{p_{i}}{ }^{o}\left(0^{-}\right)-u_{p_{i}}\right]\right) \\
\text { s.t. } & \mathbf{g}\left(\mathbf{x}, \mathbf{u}, \mathbf{s}\left(0^{-}\right)\right)=\mathbf{0} \\
& \mathbf{u}_{\mathbf{r}}^{\min } \leq \mathbf{u}_{\mathbf{r}} \leq \mathbf{u}_{\mathbf{r}}{ }_{\max } \\
& \operatorname{Pr}\left(\mathbf{u}_{\mathbf{p}}^{\min } \leq \mathbf{u}_{\mathbf{p}} \leq \mathbf{u}_{\mathbf{p}}{ }^{\max }\right) \geq \alpha \\
& \mathbf{V}^{\min } \leq \mathbf{V}\left(\mathbf{x}, \mathbf{u}_{\mathbf{r}}, \mathbf{u}_{\mathbf{p}}\right) \leq \mathbf{V}^{\max } \\
& \mathbf{Q}^{\min } \leq \mathbf{Q}\left(\mathbf{x}, \mathbf{u}_{\mathbf{r}}, \mathbf{u}_{\mathbf{p}}\right) \leq \mathbf{Q}^{\max } \\
& \mathbf{P}_{p f}^{\min } \leq \mathbf{P}_{p f}\left(\mathbf{x}, \mathbf{u}_{\mathbf{r}}, \mathbf{u}_{\mathbf{p}}\right) \leq \mathbf{P}_{p f}^{\max } \\
& \mathbf{P}_{p f}\left(\mathbf{x}, \mathbf{u}_{\mathbf{r}}, \mathbf{u}_{\mathbf{p}}\right)=\mathbf{P}_{p f}^{o}-\frac{1}{\mathbf{R}} \Delta F
\end{array}
$$

where $\mathbf{x}$ is the state vector, $\mathbf{u}_{\mathbf{r}}$ and $\mathbf{u}_{\mathbf{p}}$ are the vectors of $n_{r}$ and $n_{p}$ controlled variables, respectively. $\mathbf{u}_{\mathbf{r}}$ may be either decreased or increased such as generator voltage setpoints, while $\mathbf{u}_{\mathbf{p}}$ represents controlled variables constrained to be changed in one direction only, such as load shedding. $u_{r i}{ }^{o}\left(0^{-}\right)$ and $u_{p_{i}}{ }^{\circ}\left(0^{-}\right)$denote the pre-disturbance value of $\mathbf{u}_{\mathbf{r}}$ and $\mathbf{u}_{\mathbf{p}}$, respectively, and $c_{i}$ reflects the respective costs of the various controls. Vector $\mathbf{V}$ represents bus voltage magnitudes, $\mathbf{Q}$ is the vector of reactive power productions, and $\mathbf{P}_{p f}$ represents active power of generators participating in primary frequency control. Constraint ( $3 b)$ models the steady-state operation of the system in the post-disturbance post-control configuration. Equation ( $3 \mathrm{~h}$ ) denotes distributed slack bus model, where $\mathbf{P}_{p f}^{o}$ is the base generation, $\mathbf{R}$ is the speed droop of generators, and $\Delta F$ is the frequency deviation. In the optimization problem (3), the uncertainty appears in objective function (3a) and the set of constraints in (3d).

Several points need to be addressed regarding the control scheme.

1) Load Power Restoration: As was shown in [3], anticipating load power restoration resulted by LTCs actions, instead of relying on detailed load response model, can lead to a satisfactory voltage control. In this work, the impact of automatic LTCs is incorporated by setting the load powers to $\mathbf{s}\left(0^{-}\right)$which represents pre-disturbance load value.

2) Controller Activation: As noted in Section II, the controller receives measured "snapshots" of voltage and bus power injections at a 10-s sample period. The controller issues no changes in control variables while all of the bus voltage magnitudes and reactive power generations are within prescribed limits. Upon a detection of unacceptable voltages or generator reactive powers, the controller computes the changes required in control variables to bring all the measured values back to acceptable ranges. In addition, the network topology, if changed after the disturbance, is updated in the controller. A 10-second delay is also considered to implement control actions on system, in order to consider computational time and communication delays between the controller and generator/load sites. Note that, since the solution to the OPF formulation (3) provides a 
static steady-state operating point to be reached by the system, there is no need to repeat (3) in a time sequence or receding horizon.

3) Generator Voltage Setpoints: The terminal voltages of many generators are different from their AVR setpoints because of the existence of steady-state error [2]. In (3), it is desired to find the new AVR setpoints of generators while the terminal voltage of the machines is involved in the optimization. To deal with this problem, and knowing that the change in AVR setpoint results in almost the same change in the terminal voltage of the machine, the controller provides setpoint "corrections" of AVRs based on desired changes in terminal voltages.

4) Generators Reactive Powers: According to generator capability curve, the maximum reactive power limit of each generator is a function of its associated active power generation and terminal voltage. Therefore, $\mathbf{Q}^{\max }$ is calculated based on received snapshots of bus voltages and active power generation utilizing [2. eqs. 3.32a, 3.32b, and 3.49], where the effect of saturation is neglected for the sake of simplicity. The equations are given in the Appendix.

The actions of OELs limiting the field current of generators need to be incorporated in the controller since they prevent generators from increasing their voltages. However, in practice, it is rarely possible to have the information about the field current profiles, or the action of OELs in the control center. Instead, using the snapshot of a generator reactive power, it is likely that the field current of a generator becomes limited by the action of its OEL when the sampled reactive power is equal or higher than its $\mathbf{Q}^{\max }$. Therefore, no increase in voltage of generators exceeding their maximum reactive power is allowed in (3).

\section{B. CCO Solution Approach}

The solution to optimization problem (3) is required to be feasible and able to stabilize the system within a preassigned boundary of probability $\alpha \pm \Delta \alpha$. The joint probabilistic constraint (3d) is a function of multirandom variables that is transformed to a set of deterministic constraints. As described in Section II-B, the upper bound of the joint constraint (3d) can be written as separated equivalent deterministic equations such that

$$
u_{p_{j}} \leq \mu_{j}+\sigma_{j} Z_{\alpha} j=1, \ldots, K
$$

where $\mu_{j}$ and $\sigma_{j}^{2}$ are the mean and variance of the $j$ th curtailable demand with normal distribution. $Z_{\alpha}$ could be interpreted as the loading level, which is equal to $\phi^{-1}\left(\frac{1-\alpha}{K}\right)$. The same approach can be used to convert the lower bound in (3d) to the individual deterministic constraints, which results in the following equations:

$$
u_{p_{j}} \geq \beta\left(\mu_{j}-\sigma_{j} Z_{\alpha}\right) j=1, \ldots, K
$$

where $\beta$ is the fraction of desired load to be not shed, in other words $(1-\beta)$ denotes the maximum fraction of load allowed to be curtailed. Note that, care must be taken into account when choosing $\beta$, since relatively high values of $\beta$ would lead to the load shedding lower bound in (5) to be higher than the upper bound in (4) and, consequently, infeasibility of the problem.

The objective function (3a) is transformed to the equivalent deterministic one using the E-model [24], where $u_{p}^{o}\left(0^{-}\right)$is replaced with $\mu_{j}, j=1, \ldots, K$.

For solving the CCO problem, the following three steps are performed iteratively.

Step 1) Problem (3) is solved with the equivalent deterministic objective function and the constraints $(3 \mathrm{~d})$ on load shedding replaced with (4) and (5) for a fixed scalar $Z_{\alpha}$ to determine the set of new controlled variables.

Step 2) The LHS-CD technique is used to efficiently sample the random variables to determine if the specified confidence level has been reached. For each sample taken, new AVR setting of generators, and the amount of load on curtailable buses are updated by the solution found in step 1). Although a dynamic model of system is needed to completely ensure the effectiveness of the derived solution with respect to the randomness in demand, this paper proposes a steady-state model of the system in which the feasibility of the solution is examined. The feasibility model can be described as finding a feasible operating point for the set of following equations:

$$
\begin{aligned}
\mathbf{g}\left(\mathbf{x}, \mathbf{s}\left(0^{+}\right)\right) & =\mathbf{0} \\
\mathbf{V}^{\min }\left(\mathbf{V}_{\text {ref }}\right) & \leq \mathbf{V}(\mathbf{x}) \leq \mathbf{V}^{\max }\left(\mathbf{V}_{\text {ref }}\right) \\
\mathbf{Q}^{\min } & \leq \mathbf{Q}(\mathbf{x}) \leq \mathbf{Q}^{\max } \\
\mathbf{P}_{p f}^{\min } & \leq \mathbf{P}_{p f}(\mathbf{x}) \leq \mathbf{P}_{p f}^{\max } \\
\mathbf{P}_{p f}(\mathbf{x}) & =\mathbf{P}_{p f}^{o}-\frac{1}{\mathbf{R}} \Delta F
\end{aligned}
$$

where constraint (6a) models the steady-state operation of the system, and $\mathbf{s}\left(0^{+}\right)$describes the system loading level after considering the derived load shedding. $\mathbf{V}^{\min }\left(\mathbf{V}_{\text {ref }}\right)$ and $\mathbf{V}^{\max }\left(\mathbf{V}_{\text {ref }}\right)$ in (6b) are the minimum and maximum terminal voltage of generators corresponding to the AVR setpoints, respectively. Constraints (6c) represent reactive power limits, and (6d) and (6e) are to model distributed slack bus as commented in Section III-A. The terminal voltage limits are calculated based on the new AVR setpoint, active power generation and minimum and maximum reactive power generation using [2, eqs. 3.32a, 3.32b, and 3.44] (see the Appendix) neglecting the effect of machine saturation. The new AVR setpoints can be computed by adding the default setpoints to the terminal voltage corrections determined in step 1). In order to avoid adding complexity to the model, $\mathbf{Q}^{\min }$ and $\mathbf{Q}^{\max }$ in (6c) are considered to be the static limits of reactive powers. Another option is to incorporate the maximum reactive limit equations (i.e., [2, eqs. 3.32a, 3.32b, and 3.49]) along with the equations for terminal voltage of generators based on the AVR setpoint (i.e., [2, 
eqs. 3.32a, 3.32b, and 3.44]) directly into (6). However, using this approach was not observed to have significant improvement on results other than increasing the computational burden of solving the equations.

Finally, by dividing the number of feasible cases over $N_{\text {trial }}$ samples, the probability of feasibility, $P_{\text {feas }}$, is determined.

Step 3) If $\left|\alpha-P_{\text {feas }}\right| \leq \Delta \alpha$ then the algorithm is stopped; else $Z_{\alpha}$ is updated and the previous steps are repeated. The method for updating $Z_{\alpha}$ is based on interpolation between an upper and lower bound value of $Z_{\alpha}$, and continue this procedure with replaced values for the upper or lower bound until the final value satisfying the stopping criterion is reached. The interpolation method can be found in more detail in [26].

Compared with the SSO approach presented in [3], both papers rely on the steady-state system model to optimize the control actions. However, no uncertainty is modeled in the optimization problem in [3]. In the proposed approach, the objective function includes a linear term corresponding to uncertain variables. In addition, the set of constraints (3d) incorporates uncertainty on the available load for shedding, where this is not directly considered in [3].

\section{Simulation Results}

\section{A. Test System and Simulation Data}

The proposed control scheme is implemented on Nordic32 test system [34] shown in a single-line diagram in Fig. 1. This system contains 52 buses and 20 synchronous machines (specified as $\mathrm{g}$, followed by generation number). A detailed dynamic model of generators, AVRs, governors, and load is considered to ensure employing the controller in realistic conditions. For each generator, the model includes [34]-[36]: a standard synchronous machine model with three or four rotor windings, a simple governor for generators in the North and Equiv areas (the other generators do not participate in frequency control), and a simple automatic voltage regulator including an over-excitation limiter. The synchronous machines $\mathrm{g} 1-\mathrm{g} 5$, g8-g12, and g19-g20 are hydro units with the speed droops of 0.08 p.u. on machine basis for g19 and g20 and 0.04 p.u. for the rest of the hydro units. Each load is fed through a transformer with automatic load tap changer. There is a delay of 30 seconds on the first tap change and a shorter delay on the subsequent steps. Each load is represented by an exponential model with exponent 1 (constant current) for the active power and exponent 2 (constant admittance) for the reactive power. More details on the machine parameters and load models can be found in [35], [36]. The model was simulated with MATLAB/SIMULINK using a variable step-size method to simulate its dynamics, and the optimization problem was solved using GAMS-IDE with the IPOPT solver interfaced with SIMULINK through MATGAMS [3]. The simulations were executed on a Windows machine equipped with Intel $2.00-\mathrm{GHz}$ CPU and $2 \mathrm{~GB}$ of RAM.

The proposed controller is able to change 20 generator voltages in the range [0.95 1.07] pu, and the loads at buses 1022,

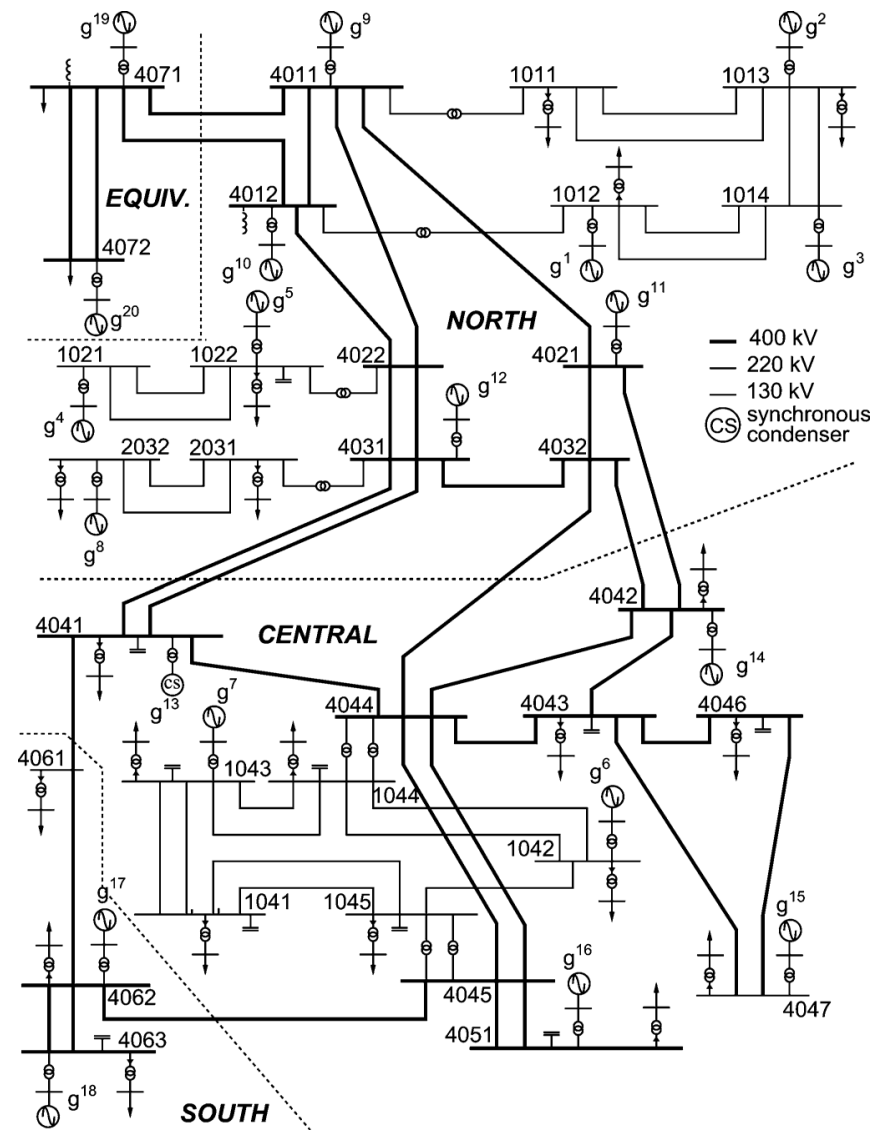

Fig. 1. One-line diagram of Nordic32 test system [34].

$1041,1042,1043,1044,1045$, and 2031. Only the distribution of the load at the seven buses is known to the controller, which is a normal distribution where there is $20 \%$ maximum error in the mean value, and no correlation among random variables. The associated costs related to the controls are: $10^{0}$ for load shedding and $10^{-3}$ for generator voltages. A maximum rate of control changes, (3c), is specified on generator voltages equal to $0.05 \mathrm{pu}$. The tolerance in probability of feasibility, $\Delta \alpha$, was considered to be 0.01 .

\section{B. Sampling Technique}

The performance of LHS-CD was compared with LHS-RP and Monte Carlo with SRS sampling to demonstrate the effectiveness of LHS-CD. In addition, the number of LHS-CD samples required to ensure satisfying the specific confidence level is determined. This analysis was based on running the feasibility model off-line with different number of samples for solutions derived from the equivalent deterministic model described in Section III-B.

It was assumed that the results obtained from 50000 Monte Carlo with SRS runs are accurate enough to be used to calculate the error of different sampling methods. Fig. 2 illustrates the average error in standard deviation of probability of feasibility repeated 20 runs for different sampling methods and sampling numbers. The average error in mean value of probability of feasibility was found to be less than $0.7 \%$ for all three sampling techniques and, therefore, is not shown. As seen in Fig. 2, the 


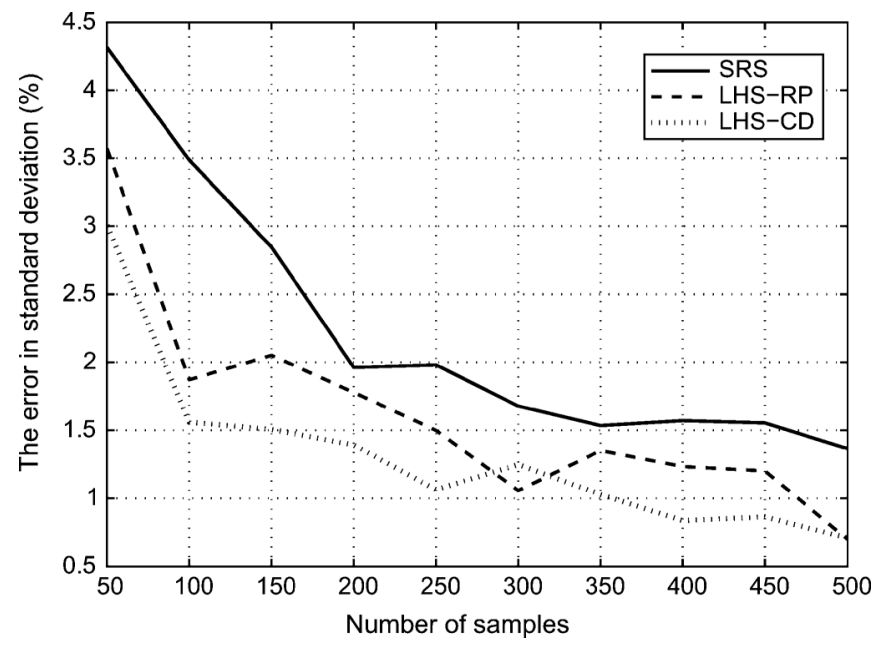

Fig. 2. Error in standard deviation of probability of feasibility for different sampling methods with respect to sampling numbers.

LHS-CD achieves a better performance in terms of produced error compared with SRS and LHS-RP.

In addition, it can be observed that with 100 samples the probability of feasibility could be achieved with an error in standard deviation of approximately $1.5 \%$ which is the almost around the accepted tolerance in $\Delta \alpha$. For the above reason, 100 samples generated by the LHS-CD method are used in all tests reported hereafter.

The requirement for the number of samples was verified after running offline simulations for different operating conditions. Although different operating conditions might affect the convergence of the equations and consequently the number of samples, a significant difference for the case of Nordic32 system was not found. In addition, the solution derived with the proposed control scheme was shown to be conservative in terms of the chance of system saving allowing some compromise in terms of number of samples. The number of samples for other systems can be determined by a similar approach.

\section{Stabilization of an Unstable Scenario}

A disturbance case was considered where the outage of transmission line 4032-4044 (Fig. 1) happens at $t=20 \mathrm{~s}$. As the result of actions of automatic LTCs trying to restore the load, and the field current limitation of $\mathrm{g} 6, \mathrm{~g} 7, \mathrm{~g} 11, \mathrm{~g} 12$, g13, $\mathrm{g} 14, \mathrm{~g} 15$, and $\mathrm{g} 16$, the system collapse happened at $t=136 \mathrm{~s}$. Using the deterministic controller with the uncertain loads represented by their mean values, resulted in system collapse at $t=429 \mathrm{~s}$. Although the controller expected to stabilize the system by shedding 123.7 MW load, it failed due to the actual loads being different than the expected values. The actual amounts of curtailable loads in this case were $(102,107$, $104,107,92,102,95) \%$ of the mean value. The CCO with $\alpha=0.9$ was implemented in the controller, and it was able to stabilize the system for the described case, with 314-MW load shedding. The voltage profile of bus 1044 is shown in Fig. 3 for three cases of: without controller, deterministic controller, and stochastic controller. Note that, incorporating the "actual" amount of load, i.e., considering perfect information about

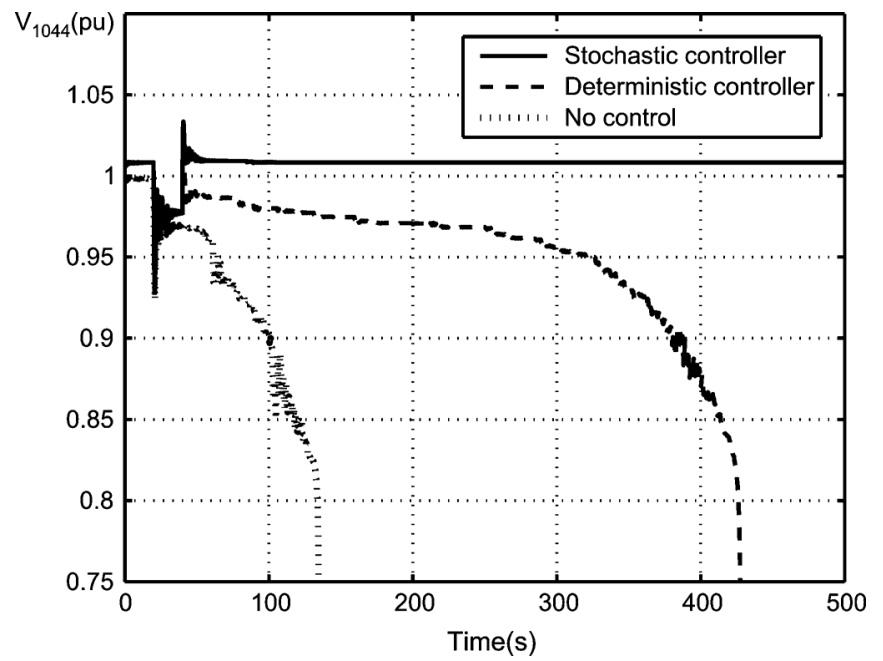

Fig. 3. Voltage at bus 1044 without controller, with the deterministic and stochastic controller.

the load available for shedding, in the deterministic controller resulted in $192 \mathrm{MW}$ of load shedding.

The above simulation, with $\alpha=0.9$, was performed with 100 samples of the initial load at curtailable load buses, which resulted in 91 stabilized cases. In the successful cases, the amount of the curtailed load was almost the same with an average of $308 \mathrm{MW}$ and 7-MW standard deviation. As can be seen, the controller was observed to be slightly more conservative in satisfying the desired confidence level compared to the actual response of the system. However, the steady-state feasibility model can still provide a satisfactory solution in a reasonable time without incorporating dynamic system equations. By using the deterministic controller with the mean value of uncertain loads, it was found that the controller was able to stabilize the system only in 48 cases, therefore, the value of using the stochastic controller is to enhance voltage stability of the system by the factor of $90 \%$.

For the above set of simulations, the values of $\beta$ which led into feasibility of (4) and (5) were found to be greater than 0.72 . This value can be calculated based on the minimum value of $Z_{\alpha}$ to be used in the CCO iterative approach. For those values of $\beta$, it was observed that (5) is not a binding constraint on the optimization problem. Similar results were found for the rest of simulations described hereafter.

\section{Stabilization of a Low-Profile (Stable) Scenario}

A low-profile but long-term stable voltage scenario was addressed with the same transmission line outage occurrence where the load in Central area (see Fig. 1) is initially lower. Similar to Section IV-C, the actual amounts of curtailable loads were $(102,107,104,107,92,102,95) \%$ of the mean value. As illustrated in Fig. 4 with dotted curve, voltage at bus 1041 fell below the minimum limit of 0.95 . Using the deterministic controller with the mean value of load at curtailable buses did not improve the situation, as shown by the dashed curve in Fig. 4. The stochastic controller with $\alpha=0.9$ was able to restore all bus voltages within the acceptable range by adjusting generator voltages and $199 \mathrm{MW}$ of load shedding. 


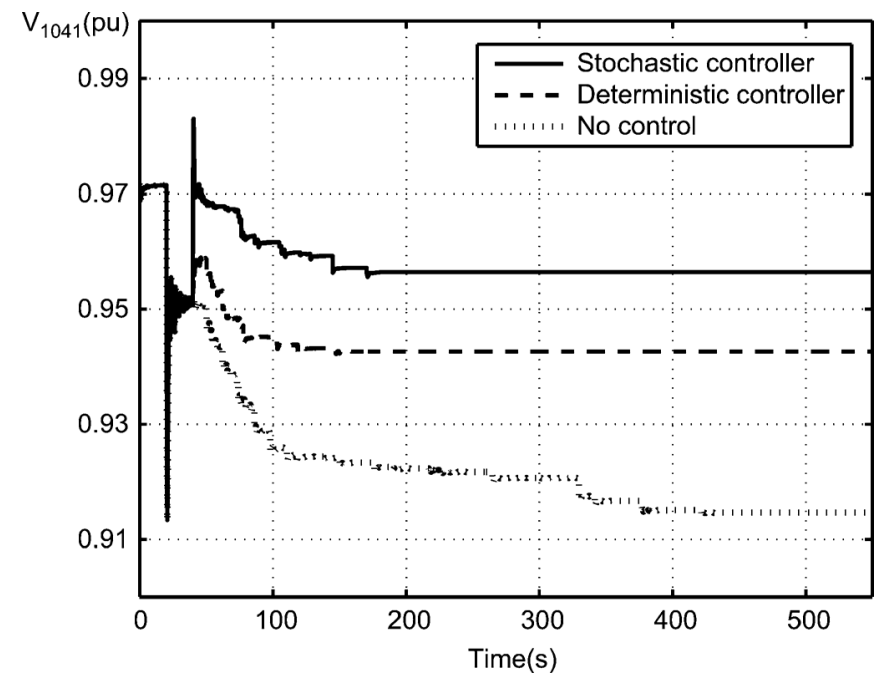

Fig. 4. Voltage at bus 1041 without controller, with the deterministic and stochastic controller.

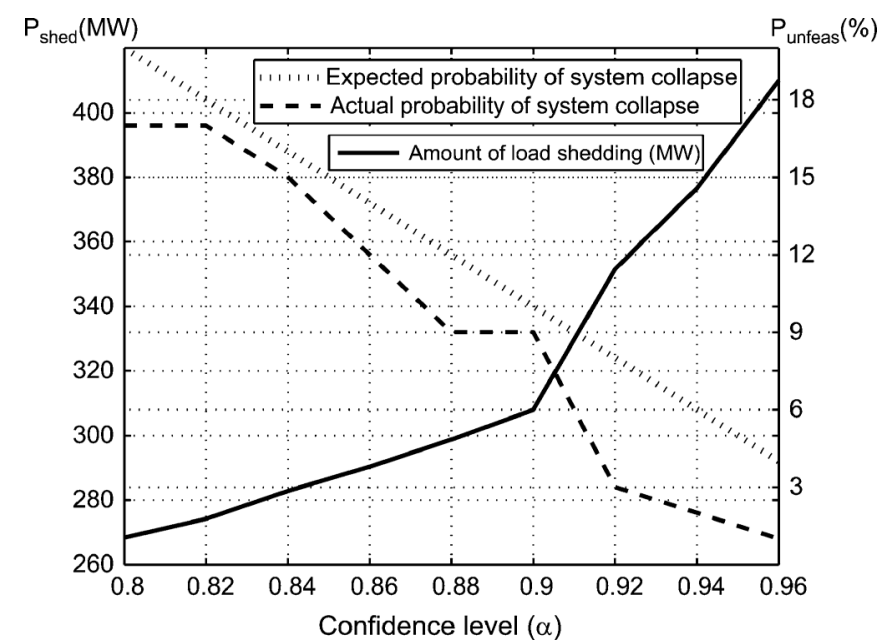

Fig. 5. Amount of load shedding, the expected chance of system collapse by the controller, and actual chance of system collapse $\left(P_{\text {unfeas }}\right.$ denotes the probability of system collapse).

\section{E. Sensitivity Analysis}

Here, the sensitivity of the results to the confidence level and maximum error in the load available for shedding is analyzed. The results of load shedding, presented in this section, are the average values based on running the simulations with 100 samples of initial load on curtailable buses. In all scenarios, the iterative algorithm was converged to a solution.

At first, the impact of different confidence levels on the results was investigated as illustrated in Fig. 5. In this figure, the amount of load shedding, the expected chance of system collapse and the actual chance of system collapsed are presented. As can be observed, lower confidence levels lead to lower amount of load shedding with the compromise of increasing the chance of system failure, and vise versa. The amount of load shedding increases rapidly from $\alpha=0.92$ with the advantage of higher chance in system stabilization. In addition, the controller is conservative for the all values of $\alpha$ with the most conservative solution for $\alpha=0.92$ which resulted in actual chance of system stabilization being as $97 \%$.
In the final set of simulations, the effect of changing the maximum error in the load on curtailable buses was verified. The level of confidence level was selected to be $\alpha=0.9$ in this set of results. Increasing the maximum error in load led to higher amount of load shedding. The maximum error in the load was increased from $10 \%$ to $30 \%$ of the mean value, which resulted in 200-370-MW average curtailed load. The number of stabilized cases was above 91 for all different values of maximum errors, ranging from 91 to 93 cases.

\section{F. Computational Burden}

The computational burden of the proposed controller is composed of the time required for the iterative procedure of solving optimization problem (3) and feasibility model (6). In the Nordic32 test system, the problem (3) contains 124 equality constraints, up to 129 inequality constraints, 103 state variables, and up to 27 controls. The feasibility model (6) involves 124 equality constraints, 94 inequality constraints, and 103 state variables. The computational burden of the proposed controller is mainly due to the iterative approach needed to be performed to find the solution.

Problems of large dimension may require specific computing structures, such as parallelism and multicore machines to achieve computational times appropriate for real-time applications. Problems of moderate dimension should be efficiently solved using sequential processors (including the possibility for model reduction by considering lower dimension problem of specific part of the system with full exploitation of the problems structure such as sparsity).

With respect to adopting data parallelism for large dimension problems [37], solving the feasibility model (6) for different samples is an independent task that can be distributed over several machines. Therefore, the total computational time in each iteration is the summation of simulation time of optimization problem, found to be $0.01 \mathrm{~s}$, and the maximum time spent on running the feasibility model for each sample, which is $0.25 \mathrm{~s}$. The higher computational time of feasibility model compared with the optimization problem is due to infeasible cases that might happen in the former and CPU time consumed till the maximum number if iterations is reached. Since no communication is required between individual threads, communication time can be neglected in this regard [38].

\section{CONCLUSION}

A CCO was proposed in order to consider online voltage control where uncertainty is involved in available load for shedding. A steady-state model was developed to ensure satisfying the given confidence level with the solution found by the equivalent deterministic problem. The LHS-CD technique was employed to efficiently sample from normally distributed load, and was shown to be effective compared with the SRS, and LHS-RP techniques. Scenarios with unstable voltage, and low but stable voltage profiles were considered to show the effectiveness of the proposed controller in different situations. Using the steady-state model to verify the feasibility of the derived solution was shown to lead to acceptable results. Sensitivity analysis around the confidence level and the maximum error in 
curtailable loads was conducted. Finally, a discussion on the computational burden of the proposed controller was provided.

\section{APPENDIX}

Using [2, eqs. 3.32a, 3.32b, 3.44, and 3.49] in this paper to calculate maximum reactive power of generators and terminal voltage limits as a function of AVR setpoints yields

$$
\begin{aligned}
P= & \frac{E_{q} E_{q}^{s} V}{X_{l} E_{q}+\left(X_{d}-X_{l}\right) E_{q}^{s}} \sin (\delta-\theta) \\
& +\frac{E_{q} V^{2}}{2}\left(\frac{1}{X_{l} E_{q}+\left(X_{q}-X_{l}\right) E_{q}^{s}}\right. \\
& \left.-\frac{1}{X_{l} E_{q}+\left(X_{d}-X_{l}\right) E_{q}^{s}}\right) \sin 2(\delta-\theta) \\
Q= & \frac{E_{q} E_{q}^{s} V}{X_{l} E_{q}+\left(X_{d}-X_{l}\right) E_{q}^{s}} \cos (\delta-\theta) \\
& -E_{q} V^{2}\left(\frac{\sin ^{2}(\delta-\theta)}{X_{l} E_{q}+\left(X_{q}-X_{l}\right) E_{q}^{s}}\right. \\
& \left.+\frac{\cos ^{2}(\delta-\theta)}{X_{l} E_{q}+\left(X_{d}-X_{l}\right) E_{q}^{s}}\right) \\
E_{q}= & G\left(V_{\mathrm{ref}}-V\right) \\
E_{q}= & E_{q}^{\lim }
\end{aligned}
$$

where $E_{q}$ is the open-circuit voltage of machine, $E_{q}^{s}$ is the saturated open-circuit voltage, $X_{d}$, and $X_{q}$ are direct and quadrature axis synchronous reactance, respectively, $X_{l}$ is the leakage reactance, $\delta-\theta$ is the internal angle. $V_{\text {ref }}$ and $G$ are the AVR setpoint and AVR gain of the machine, respectively. $P, Q$, and $V$ are the machine active power, reactive power, and terminal voltage, respectively.

\section{REFERENCES}

[1] C. W. Taylor, Power System Voltage Stability. New York: McGrawHill, 1994.

[2] T. Van Cutsem and C. Vournas, Voltage Stability of Electric Power Systems. Boston, MA: Kluwer, 1998.

[3] M. Glavic, M. Hajian, W. Rosehart, and T. van Cutsem, "Receding-horizon multi-step optimization to correct nonviable or unstable transmission voltages," IEEE Trans. Power Syst., vol. 26, no. 3, pp. 1641-1650, Aug. 2011.

[4] H. Vu, P. Pruvot, C. Launay, and Y. Harmand, "An improved voltage control on large-scale power system," IEEE Trans. Power Syst., vol. 11, no. 3, pp. 1295-303, 1996.

[5] D. Popovic, V. Levi, and Z. Gorecan, "Co-ordination of emergency secondary-voltage control and load shedding to prevent voltage instability," Proc. Inst. Electr. Eng.-Gen., Trans. Distrib., vol. 144, no. 3, pp. 293-300, 1997.

[6] M. Larsson and D. Karlsson, "Coordinated system protection scheme against voltage collapse using heuristic search and predictive control," IEEE Trans. Power Syst., vol. 18, no. 3, pp. 1001-6, Aug. 2003.

[7] J. Wen, Q. Wu, D. Turner, S. Cheng, and J. Fitch, "Optimal coordinated voltage control for power system voltage stability," IEEE Trans. Power Syst., vol. 19, no. 2, pp. 1115-22, May 2004.

[8] A. Verma, A. Srividya, and H. Kumar, "A framework using uncertainties in the composite power system reliability evaluation," Electr. Power Compon. Syst., vol. 30, no. 7, pp. 679-91, Jul. 2002.

[9] L. Arya, L. Titare, and D. Kothari, "An approach to mitigate the risk of voltage collapse accounting uncertainties using improved particle swarm optimization," Appl. Soft Computing J., vol. 9, no. 4, pp. 1197-1207, 2009.
[10] K. Abdul-Rahman and S. Shahidehpour, "Application of fuzzy sets to optimal reactive power planning with security constraints," IEEE Trans. Power Syst., vol. 9, no. 2, pp. 589-97, Jul. 1994.

[11] C. Indulkar and B. Viswanathan, "Deterministic and probabilistic approach to voltage stability of series-compensated EHV transmission lines," IEEE Trans. Power App. Syst., vol. PAS-102, no. 7, pp. 2317-22, Jul. 1983.

[12] A. Saric, M. Calovic, and V. Strezoski, "Fuzzy multi-objective algorithm for multiple solution of distribution systems voltage control," Int. J. Electr. Power Energy Syst., vol. 25, no. 2, pp. 145-53, Feb. 2003.

[13] C.-L. Su, "Stochastic evaluation of voltages in distribution networks with distributed generation using detailed distribution operation models," IEEE Trans. Power Syst., vol. 25, no. 2, pp. 786-795, May 2010.

[14] A. Rodrigues, R. Prada, and M. Da Guia da Silva, "Voltage stability probabilistic assessment in composite systems: Modeling unsolvability and controllability loss," IEEE Trans. Power Syst., vol. 25, no. 3, pp. 1575-88, Aug. 2010.

[15] H. Zhang and P. Li, "Chance constrained programming for optimal power flow under uncertainty," IEEE Trans. Power Syst., vol. 26, no. 4, pp. 2417-2424, Nov. 2011.

[16] Z. Hu, X. Wang, and G. Taylor, "Stochastic optimal reactive power dispatch: Formulation and solution method," Int. J. Electr. Power Energy Syst., vol. 32, no. 6, pp. 615-21, Jul. 2010.

[17] L. Wu, M. Shahidehpour, and T. Li, "Stochastic security-constrained unit commitment," IEEE Trans. Power Syst., vol. 22, no. 2, pp. 800-11, May 2007.

[18] A. Saric, F. Murphy, A. Soyster, and A. Stankovic, "Two-stage stochastic programming model for market clearing with contingencies," IEEE Trans. Power Syst. (USA), vol. 24, no. 3, pp. 1266-78, Aug. 2009.

[19] A. T. Schwarm and M. Nikolaou, "Chance-constrained model predictive control," AIChE J., vol. 45, no. 8, pp. 1743-1752, 1999.

[20] Y. Zhang, D. Monder, and J. Forbes, "Real-time optimization under parametric uncertainty: A probability constrained approach," $J$. Process Control, vol. 12, no. 3, pp. 373-89, Apr. 2002.

[21] L. Xie, P. Li, and G. Wozny, "Chance constrained nonlinear model predictive control," Assess. Future Directions Nonlinear Model Predictive Control, vol. 358, no. 3, pp. 295-304, 2007.

[22] P. Li, M. Wendt, and G. Wozny, "A probabilistically constrained model predictive controller," Automatica, vol. 38, no. 7, pp. 1171-6, Jul. 2002.

[23] J. Valenzuela and M. Mazumdar, "Statistical analysis of electric power productions costs," IIE Trans., vol. 32, pp. 1139-1148, 2000.

[24] A. Charnes and W. Cooper, "Deterministic equivalents for optimizing and satisficing under chance constraints," Oper. Res., vol. 11, no. 1, pp. 18-39, Feb. 1963.

[25] A. Nemirovski and A. Shapiro, "Convex approximations of chance constrained programs," SIAM J. Optim., vol. 17, no. 4, pp. 959-996, 2006.

[26] U. Ozturk, M. Mazumdar, and B. Norman, "A solution to the stochastic unit commitment problem using chance constrained programming," IEEE Trans. Power Syst., vol. 19, no. 3, pp. 1589-98, Aug. 2004.

[27] M. Mazadi, W. Rosehart, O. Malik, and J. Aguado, "Modified chance-constrained optimization applied to the generation expansion problem," IEEE Trans. Power Syst., vol. 24, no. 3, pp. 1635-1636, Aug. 2009.

[28] R. Allan, A. Leite de Silva, and R. Burchett, "Evaluation methods and accuracy in probabilistic load flow solutions," IEEE Trans. Power Appar. Syst., vol. PAS-100, no. 5, pp. 2539-2546, May 1981.

[29] A. Schellenberg, W. Rosehart, and J. Aguado, "Cumulant-based probabilistic optimal power flow (p-opf) with gaussian and gamma distributions," IEEE Trans. Power Syst., vol. 20, no. 2, pp. 773-781, May 2005.

[30] M. McKay, R. Beckman, and W. Conover, "Comparison of three methods for selecting values of input variables in the analysis of output from a computer code," Technometr., vol. 21, no. 2, pp. 239-245, 1979.

[31] P. Jirutitijaroen and C. Singh, "Reliability constrained multi-area adequacy planning using stochastic programming with sample-average approximations," IEEE Trans. Power Syst., vol. 23, no. 2, pp. 504-513, May 2008.

[32] H. Yu, C. Chung, K. Wong, H. Lee, and J. Zhang, "Probabilistic load flow evaluation with hybrid latin hypercube sampling and cholesky decomposition," IEEE Trans. Power Syst., vol. 24, no. 2, pp. 661-667, May 2009.

[33] R. L. Iman and W. J. Conover, "A distribution-free approach to inducing rank correlation among input variables," Commun. Statist.-Sim. Comput., pp. 311-334, Jan. 1982. 
[34] M. Glavic and T. Van Cutsem, "Wide-area detection of voltage instability from synchronized phasor measurements. part II: Simulation results," IEEE Trans. Power Syst., vol. 24, no. 3, pp. 1417-1425, 2009

[35] M. Stubbe, "Long-term dynamics phase II, Report of CIGRE Task Force 32.02.08 1995.

[36] D. Peppas, "Development and Analysis of Nordic32 Power System Model in Powerfactory," master's thesis, School of Elect. Eng., Elect. Power Syst., Royal Inst. Technol., Stockholm, Sweden, 2008.

[37] D. E. Culler, J. P. Singh, and A. Gupta, Parallel Computer Architecture: A Hardware/Software Approach. San Francisco: Morgan Kaufmann, 1999.

[38] R. Chandra, L. Dagum, D. Kohr, D. Mayadan, J. Mcdonald, and R. Menon, Parallel Programming in OpenMP. San Francisco, CA: Morgan Kaufmann, 2001.

Mahdi Hajian (M'07) received the B.Sc. degree from the Isfahan University of Technology, Isfahan, Iran, in 2005, and the M.Sc. degree from Sharif University of Technology, Tehran, Iran, in 2007. He is currently working toward the Ph.D. degree at the Department of Electrical and Computer Engineering, University of Calgary, Calgary, AB, Canada.

His research interests are in stochastic optimizations and control of power systems.
Mevludin Glavic (M'04-SM'07) received the M.Sc. degree from the University of Belgrade, Belgrade, Serbia, in 1991, and the Ph.D. degree from the University of Tuzla, Tuzla, Bosnia, in 1997, respectively.

He was with the University of Wisconsin-Madison from 1999 to 2000 as a Fulbright Postdoctoral Scholar and with the University of Liege from 2001 to 2004 as Research Fellow, 2006-2009 as a Visiting Professor. Presently, he is an Associate Consultant with Quanta Technology, Raleigh, NC, and visiting a Researcher with the University of Liege, Liege, Belgium. His research interests are in power system dynamics, stability, control, and optimization.

William D. Rosehart (M'01-SM'06) received the B.Sc., M.Sc., and Ph.D. degrees in electrical engineering from the University of Waterloo, Waterloo, ON, Canada, in 1996, 1997, and 2001, respectively.

He has been with the Schulich School of Engineering, University of Calgary, Calgary, AB, Canada, since 2001, where he is currently a Professor with the Department of Electrical and Computer Engineering. His research interests are in optimization and stability analysis of energy systems.

Hamidreza Zareipour (SM'09) received the B.Sc. degree from K. N. Toos University of Technology, Tehran, Iran, in 1995, the M.Sc. degree from Tabriz University, Tabriz, Iran, in 1997, and the Ph.D. degree from the University of Waterloo, Waterloo, ON, Canada, in 2006, all in electrical engineering.

He currently is an Assistant Professor with the Department of Electrical and Computer Engineering, University of Calgary, Calgary, AB, Canada. His research focuses on economics, planning, and management of intelligent electric energy systems in a competitive electricity market environment. 